\title{
SEMICONDUCTORS IN PRAGUE
}

\section{A Report on the \\ Fourth International Conference on Semiconductor Physics}

\author{
by Harry C. Gatos, Lincoln Laboratory, MIT
}

The Fourth International Conference on Semiconductor Physics was held in Prague August 28 to September 2, 1960. The main topics covered were: band structure and related problems, transport phenomena, optical properties, resonance phenomena, radiation damage, excitons, photoconductivity, surface phenomena, thermal properties and thermoelectricity, ionic crystals, and semiconductor compounds.

It was a truly international conference attended by approximately 700 scientists representing many countries and all continents. Drs. Shockley, Bardeen and Brattain, the discoverers of the transistor which marked the new era in semiconductor physics were present. Of the approximately 260 papers presented, the majority came from American (110 papers) and Soviet (40 papers) laboratories.

Since the last international conference (1958), considerable progress has been made in all fronts of theoretical and experimental semiconductor physics. Transport phenomena received the greatest emphasis among the many fields of study in semiconductor physics (40 papers). As a result, the understanding of lattice scattering and electronphonon interaction was markedly advanced. A distinctly new research area emerged in the last two years: carrier tunneling in narrow $n-p$ junctions. This phenomenon was investigated recently by Esaki in Japan and led to the now well-known Esaki or tunnel diode which is an extremely fast switching, negative resistance element. Aside from its applied potential, this phenomenon provides a new approach to the study of important aspects of semiconductor physics. Significant results were reported on the effect of high magnetic fields (Lincoln Laboratory, M.I.T.) and on the effect of high pressures (Harvard University) on tunneling. Other significant reports on tunneling were from the General Electric Research Laboratory, Bell Telephone Laboratories, Hughes
Products, and IBM Research Laboratory.

The presence of helicons in semiconductors was announced in a paper from the Ecole Normale Superleure (Paris). Helicons (circularly polarized waves) are present during the propagation of electromagnetic waves in a magnetic field. The helicons or their equivalent were recognized by plasma physicists.

There were a relatively small number of papers (9) on thermal properties and thermoelectricity. As it was pointed out by A. F. Joffe, a pioneer in thermoelectricity from the Academy of Sciences in Leningrad, there are still a number of unsolved problems regarding scattering of electrons and phonons and other aspects of thermoelectricity.

Surface phenomena continue to occupy an important place in semiconductor science and technology since communication with the semiconductor bulk properties must be achieved through surfaces. However, the preparation of clean and reproducible surfaces and their characterization remains a very complex problem. Progress is being made but at a relatively slow rate. Interesting papers were presented on the theory of surface states, surface diffusion effects, conduction phenomena, recombination, work damage, chemical interactions, etc.

Elemental semiconductors (silicon and germanium) are still the basic materials in semiconductor physics studies. Their extremely high purity in crystal imperfection, together with the broad experimental and theoretical knowledge of these materials makes them particularly suitable for truly-advanced and highlyrefined studies. Nevertheless, many intensive and extensive studies have been undertaken in a large number of research laboratories toward the preparation and understanding of semiconducting compounds. There seems to be no doubt that new frontiers in semiconductor science and technology will be opened by semiconducting compounds. From the papers presented in this conference, it appears that III-V intermetallic compounds have received the greatest attention and are perhaps better understood than other classes of semiconductor compounds. In particular, a great deal of theoretical and experimental work was reported on InSb, GaAs, and GaSb.

As soon as compound semiconductors of suitable purity and perfection become available, they are promptly submitted to significant fundamental measurements. This became evident from the work reported on excitons in CdS and cyclotron resonance in $\mathrm{CdAs}_{2}$. Because of their potential application to thermoelectricity, tellurides, sellenides, sulfides, and their alloys have been extensively investigated in the last two years in many countries. However, there were reports in other classes of materials. Organic semiconductors were studied in several laboratories. An interesting paper from the Academy of Sciences (Leningrad) dealt with glass-type semiconductors: chalcogenic glass exhibits purely electrical conductivity and shows the same effects and property changes as crystalline semiconductors.

Although a useful account of the many papers on semiconducting compounds cannot be given here, it became apparient during the conference that the unavailability of compounds of suitable purity and perfection is a more serious problem. Considerable progress by materials scientists on semiconducting compounds must be realized before the theoretical and technological potential of such materials can begin to be exploited.

Like other international conferences, this one afforded many scientists from a large number of laboratories a unique opportunity for communicating and exchanging ideas first hand. Such opportunities represent a significant factor in scientific progress. The proceedings of the Conference will be published by The Czechoslovak Academy of Sciences early in 1961 . 